\title{
Eficiencia de grupos de pilotes sometidos a carga lateral
}

\author{
Alberto Jaime P. y R. Ruedas-Medina \\ División de Ingenierías Civil y Geomática \\ E-mails: ajaimep@cancun.fi-a.unam.mx,roberto.ruedas@gmail.com
}

(Recibido: noviembre de 2005; aceptado: septiembre de 2006)

\section{Resumen}

El trabajo incluye una revisión bibliográfica de estudios de comportamiento teórico y experimental de pilotes sometidos a carga lateral estática. El comportamiento exper i mental fue obtenido de pruebas de carga a escala real y proporciona una base sólida para la comprensión del problema. De dos de estas pruebas de carga se obtuvieron los datos de pilotes y suelo para hacer análisis con los métodos de la viga apoyada sobre un medio elástico, el método de curvas $p-y$ y el de elementos finitos con modelación lineal del suelo. Los cálculos numéricos se compararon con las mediciones experimentales a través de gráficas de desplazamientos y momentos flexionantes vs carga lateral. El método de curvas $p$-y proporcionó los mejores resultados y modela adecuadamente la distribución de cargas laterales y momentos flexionantes entre los pilotes de un grupo. El método de elementos finitos-elástico lineal modela parcialmente la interacción suelo-grupo y calcula aceptablemente bien la eficiencia del grupo. El método de la viga apoyada sobre un medio elástico calcula resultados semejantes al de elementos finitos y mejoran al considerar el comportamiento no lineal del suelo.

Descriptores: Grupos de pilotes, carga lateral, eficiencia, curvas $p-y$.

\begin{abstract}
Thispaperincludes abibliographical review of theoretical and experimen tal be hazior studies of piles subjected to static lateral load. The exper imen tal be hav ior was ob tained from full scale load test and it provides a solid ba sis for com par i son with nu mer ical methods of analy sis, mainly beam on elas tic foun da tion, $p$-y curves method, and fi nite el e ments with lin ear mod el ing of soil. The com puter re sults were com pared with the ex per i men tal mea surements. The p-ycurves method pro vides the best re sults, mod el ing ap pro priately the dis tribu tion of lat eral loads and bend ing mo ments of piles in a group. The lin ear fi nite el e ment method mod els par tially the in ter ac tion soil-pile group and gives a rea son able an swer of the groupefficiency. The beamonelas tic founda tion method gives resultssimilar to those usi ing the fi nite el e ment method and it im proves when non lin ear be hav ior of soil is con sid ered.
\end{abstract}

Keywords: Pile groups, lat eral load, effi ciency, $p$-y curves.

\section{Introducción}

¿Por qué analizar pilotes sujetos a carga lateral? Existen varios casos en que se producen cargas laterales sobre estructuras cimentadas sobre pilotes. Algunos ejemplos son: los ciclones que traen consigo fuertes vientos y grandes olas que impactan sobre plataformas petroleras, puentes, muelles, etc. Los sismos hacen oscilar las estructuras provocando fuerzas laterales; los 
barcos al atracar en los muelles les imponen fuerzas laterales.

En este trabajo se describen brevemente, el comportamiento teórico y experimental de pilotes con la cabeza libre, sometidos a carga lateral, mismos que se comparan con los resultados de tres diferentes métodos de análisis: método de la viga apoyada sobre un medio elástico, de curvas $p-y$ y elementos finitos. El trabajo se enfoca en la comparación de la eficiencia de grupos de pilotes con la eficiencia de un pilote aislado, así como la distribución de cargas laterales y momentos entre los pilotes de un grupo. La mayoría de las pruebas de carga a escala real han sido realizadas con pilotes con cabeza libre, y es el caso de las pruebas citadas en este texto. Por lo tanto, para poder hacer la comparación con las mediciones experimentales, en los análisis se consideró que todos los pilotes, tanto aislados como de grupo, tenían la cabeza libre.

\section{Comportamiento teórico}

Hetenyi (1946), Poulos (1971 a y b) y Randolph (1981) realizaron estudios teóricos de pilotes cargados lateralmente en los que consideraron al suelo como un medio elástico lineal e isótropo. Los tres llegan a ecuaciones sencillas y fáciles de aplicar para calcular el desplazamiento y giro en la cabeza del pilote. Sin embargo, tienen el inconveniente de que los parámetros que representan al suelo son constantes, no dependen del nivel de esfuerzos o deformación unitaria que alcance el suelo en cierto instante. Desplazamientos y giros son funciones lineales de la carga lateral $y$ momento aplicados, y tienen una forma general como la expresada en la ecuación 1.

$$
u=C_{1} H+C_{2} M
$$

en la cual $u$ es el desplazamiento o el giro, $C_{1}$ y $C_{2}$ son las constantes que involucran a los parámetros del suelo, $H$ y $M$ son la fuerza lateral y el momento aplicados en la cabeza del pilote. Las gráficas de estas ecuaciones tienen la forma general mostrada en la figura 1.

Para el análisis de grupos, Poulos y Randolph utilizan factores de interacción y el principio de superposición de efectos, válido sólo para materiales elástico-lineales. Además, consideran que la distribución de presiones y momentos flexionantes obtenidos para pilotes aislados es aplicable a los pilotes del grupo.

Poulos (1971b), al estudiar la distribución de cargas en un grupo de pilotes, encontró que se concentraban en los pilotes de las esquinas en un arreglo cuadrado de 3X3 y 4X4 pilotes, mientras que los pilotes del centro eran los que menor carga

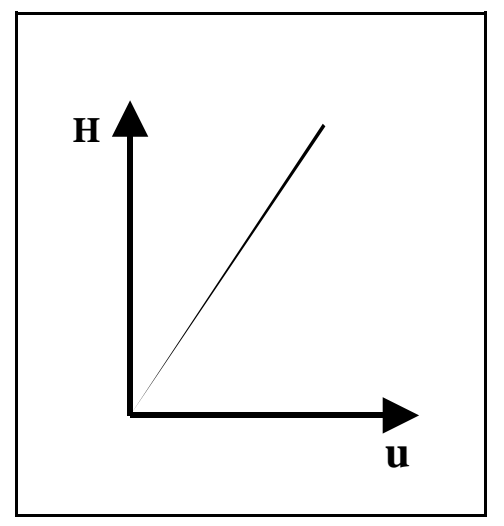

Figura 1. Forma general de la gráfica carga lateral $H$ vs desplazamiento u cuando el suelo se modela como elástico lineal 
sostenían; sin embargo, todos estos resultados difieren de los encontrados en las pruebas de carga a escala real que se comentan a continuación.

\section{Comportamiento experimental}

Brown et al. (1987 y 1988), Rollins et al. (1998) y Snyder (2004) realizaron pruebas de carga lateral en grupos de pilotes a escala real en las que encontraron que dentro del grupo los pilotes se repartían la carga en forma diferenciada: la carga que soporta cada pilote depende de la posición de la fila que lo albergue, soportando mayor carga los pilotes que están en la fila delantera y menor carga los de las filas media y trasera (ver el esquema en planta del grupo de pilotes con la carga lateral y filas delantera-FD, media-FM y trasera-FT (Figura 2).

Obtuvieron gráficas de desplazamiento en la cabeza vs carga lateral parecidas a la mostrada en la figura 2. La gráfica se puede interpretar considerando que todos los pilotes del grupo tienen el mismo desplazamiento y, como ya se había mencionado, se reparten la carga en forma diferenciada. Los pilotes de la fila delantera toman alrededor del $80 \%$ de la carga que tomaría un pilote aislado de las mismas características con el mismo desplazamiento; los pilotes de la fila media soportan alrededor del $50 \%$ y los de la fila trasera alrededor del $60 \%$ de dicha carga. También encontraron que algunas veces se intercambian el porcentaje de cargas las filas media y trasera, soportando la fila trasera la menor carga (Brownet al., 1987; Snyder, 2004).

En la figura 3, se muestran los perfiles de momentos flexionantes. En general, los autores mencionados encontraron que cuando los pilotes del grupo tienen un mismo desplazamiento, y un pilote aislado tiene la misma carga promedio del grupo, los momentos flexionantes de un pilote aislado son menores que los de cualquier

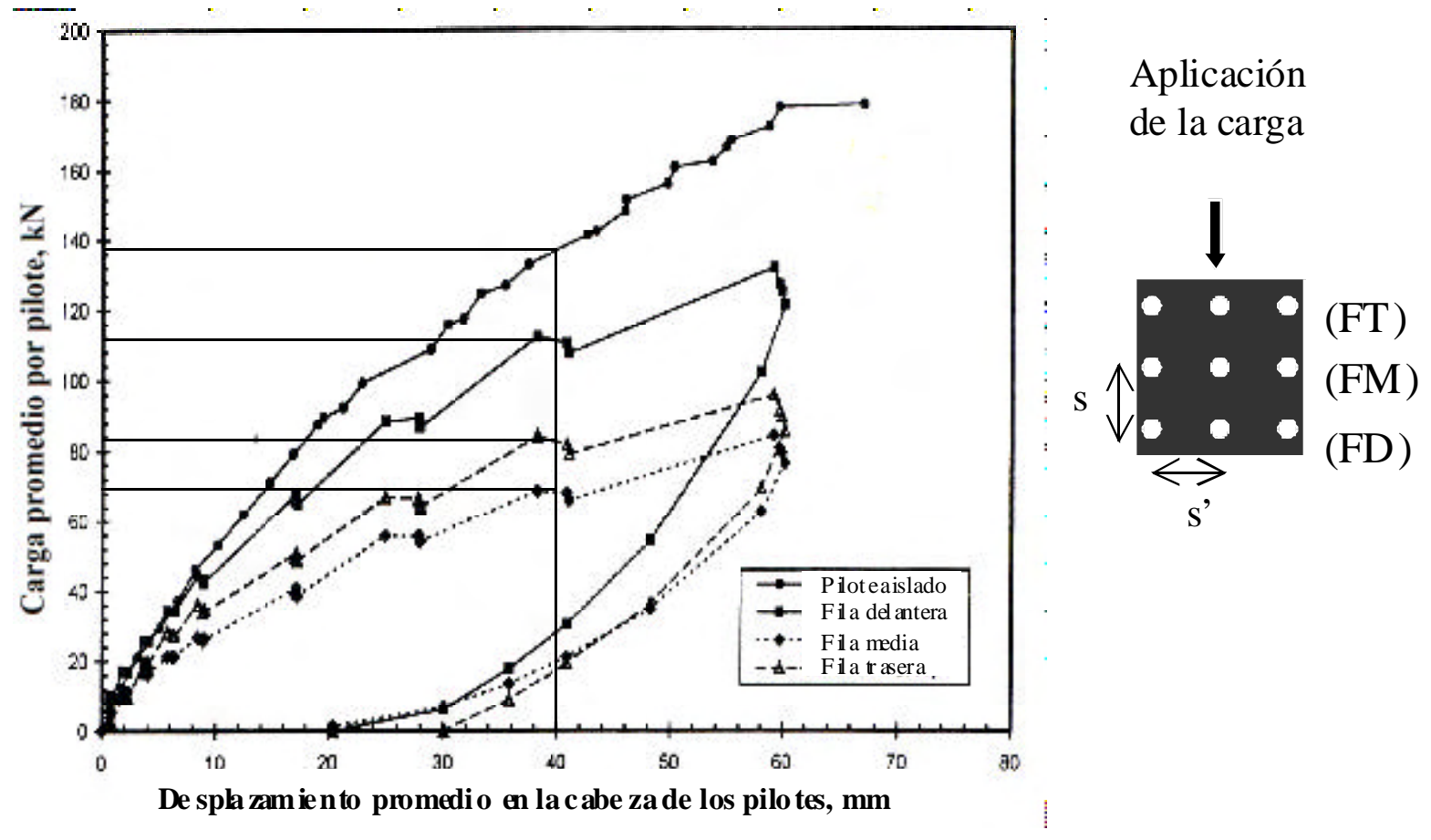

Figura 2. Prueba de carga lateral en grupo de pilotes hincados en arcilla y limo blando, Rollins et al. (1998) y esquema de grupo de pilotes con filas delantera, media y trasera. 


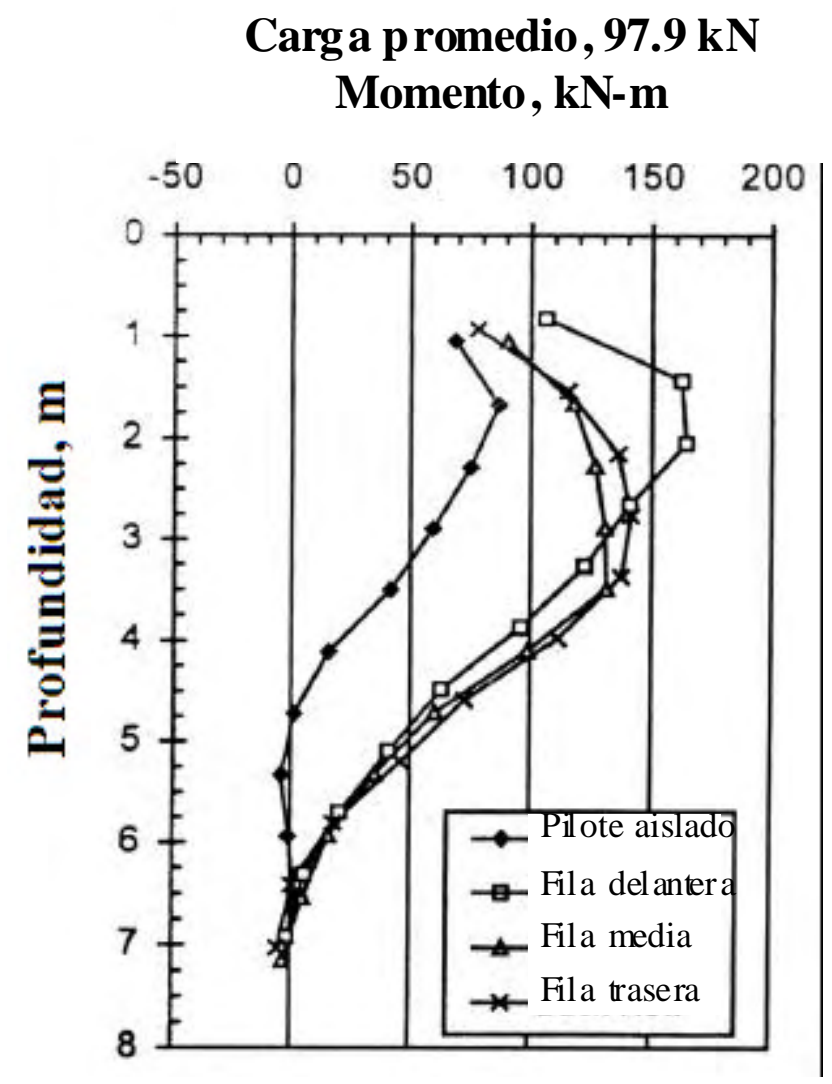

Figura 3. Perfiles de momentos flexionantes de la prueba, Rollins et al. (1998)

pilote del grupo. Los momentos flexionantes del pilote aislado son entre $30 \%$ y $50 \%$ menores que los de la fila delantera; mientras que los de las filas media y trasera son alrededor de $30 \%$ menores en los primeros metros, después alcanzan valores similares.

Además de la distribución diferenciada de cargas y momentos entre las filas de pilotes, las gráficas nos muestran un comportamiento no lineal, diferente de los resultados de análisis que consideran el suelo como elástico lineal.

Pruebas realizadas en modelos de pilotes en grupos de 2, 4, 9 y 16 (Ilyas et al., 2004) mostraron que la eficiencia de los grupos depende del nivel de desplazamiento alcanzado, de la separación entre pilotes y del número de pilotes en el grupo. La eficiencia $\eta$ la definieron como la carga lateral promedio del grupo dividido por la carga de un pilote aislado cuando tienen el mismo desplazamiento. Con separaciones centro a centro equivalentes a 3 diámetros, $\eta$ disminuye bruscamente de $100 \%$ para el pilote aislado a poco menos de $50 \%$ para grupos de 9 pilotes, considerando un desplazamiento de medio diámetro (Figura 4). Al parecer, la eficiencia tiende a un límite de $50 \%$ y se mantiene constante para grupos con 9 o más pilotes con separaciones de $3 \mathrm{D}$. También podemos ver que $\eta$ disminuye al aumentar el nivel de desplazamiento que tenga el grupo. Además, la eficiencia es mucho más alta cuando la separación entre pilotes es de 5D. 
Jaime-Paredes A. y Ruedas-Medina R.

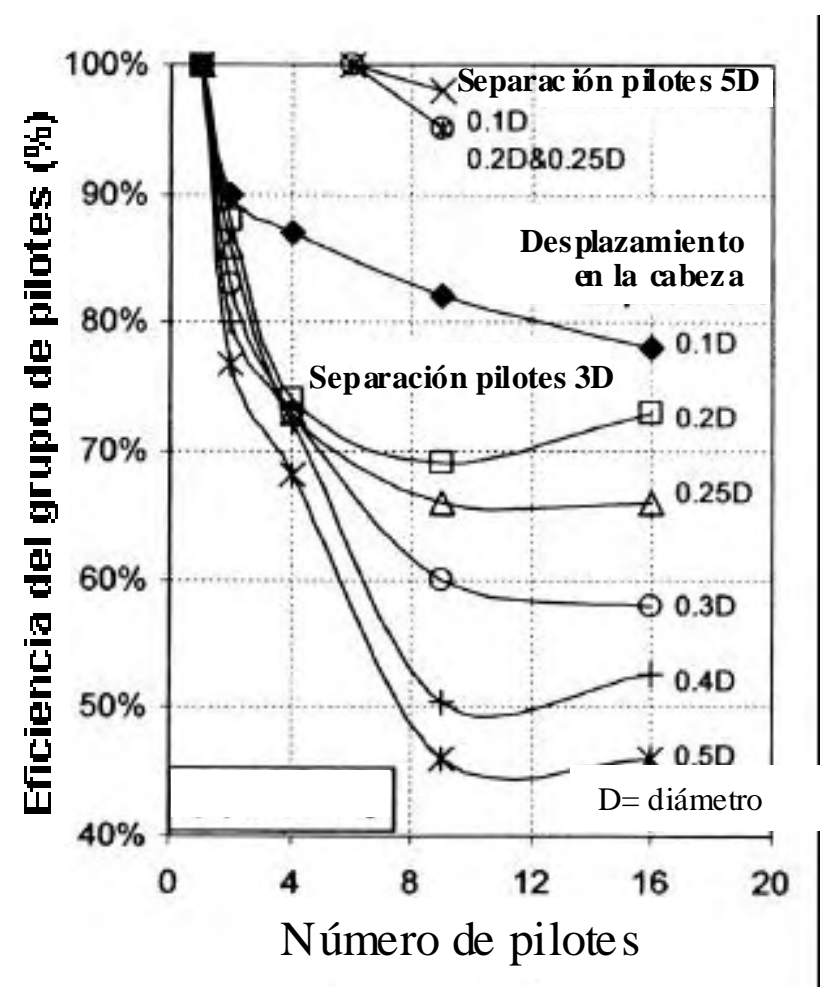

Figura 4.Eficiencia en los grupos de pilotes, Ilyas et al. (2004)

\section{Métodos de análisis}

Para estudiar el comportamiento de grupos de pilotes bajo carga lateral se usaron tres diferentes métodos de análisis. El método de la viga apoyada sobre un medio elástico, el método decurvas $p-y$, y el método de elementos finitos. Una descripción completa de los métodos se puede encontrar en Bowles (1974), Wang y Reese (1993), y Li et al. (1992), que por motivos de espacio no se incluye en este texto.

Con base en el método de la viga apoyada sobre un medio elástico, Bowles (1974) elaboró un programa en el que el suelo es modelado por medio de resortes tipo Winkler (independientes entre sí), cuyas constantes se calculan a partir del módulo de reacción $k_{s}$.

El método de curvas $p-y$ resuelve con diferencias finitas la ecuación diferencial de una viga cargada vertical y horizontalmente; el suelo es representado también por resortes tipo Winkler pero con la diferencia de que no son lineales. Dichos resortes ejercen una fuerza lateral sobre el pilote representando la reacción del suelo $P$ que depende del desplazamiento $Y$ del suelo, o en pocas palabras, las curvas $p-y$. En este estudio se usó el programa COM624P (Wang y Reese, 1989) que emplea el método de curvas $p-y$.

El método de elementos finitos divide el cuerpo en estudio en pequeñas partes llamadas elementos finitos, mismos que pueden tener diferentes formas. El programa usado en este trabajo se llama Tridimensional Estático-TEST (Li et al., 1992) y utiliza elementos sólidos hexaedros de ocho nodos, que sólo para el caso particular de este artículo se les asignaron características elásticas lineales para modelar el suelo a través de los parámetros módulo de elasticidad, relación de poisson 
y peso específico. Los pilotes son modelados a través de elementos lineales de dos nodos. En los tres métodos, los pilotes son modelados como vigas y sus parámetros son el módulo de elasticidad, relación de poisson, peso específico, área transversal y momentos de inercia.

\section{Análisis de pilotes cargados lateralmente}

Con base en los datos de pilote y suelo proporcionados por Matlock (1970) y Snyder(2004) se realizaron los análisis de pilotes aislados y grupos de pilotes, usando los programas antes mencionados para una comparación con los resultados experimentales.

\section{Resultados de los análisis}

Por razones de espacio se muestran sólo algunas de las gráficas obtenidas de los análisis, pero son representativas de los resultados generales. En las figuras 5 y 6 podemos observar los perfiles de momentos flexionantes y desplazamientos laterales experimentales de la prueba de Matlock (1970), así como los resultados de los análisis con los tres métodos. El método de curvas $p-y$ es el que mejores resultados proporciona al ajustarse notablemente bien a los perfiles experimentales.

El programa de Bowles y el programa de elementos finitos, modelando el suelo como elástico lineal, no representan adecuadamente el comportamiento de los pilotes pues se acercan a los resultados experimentales sólo cuando la carga lateral esta dentro de un estrecho rango (Figura 6). Aunque se localizan a prácticamente la misma profundidad que los experimentales, los momentos máximos calculados tienen un comportamiento similar a los desplazamientos y se alejan de los experimentales cuando se aplica una carga muy baja o muy alta. Además, a profundidades mayores a las que ocurre el momento máximo, los calculados se separan más de los experimentales.

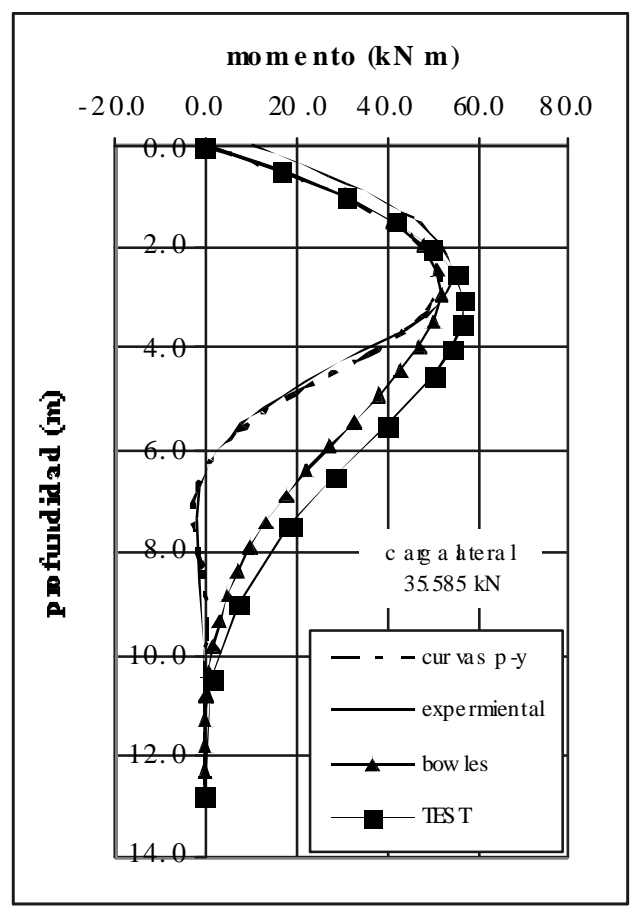

Figura 5. Perfil de momentos flexionantes de la prueba de Matlock (1970) 


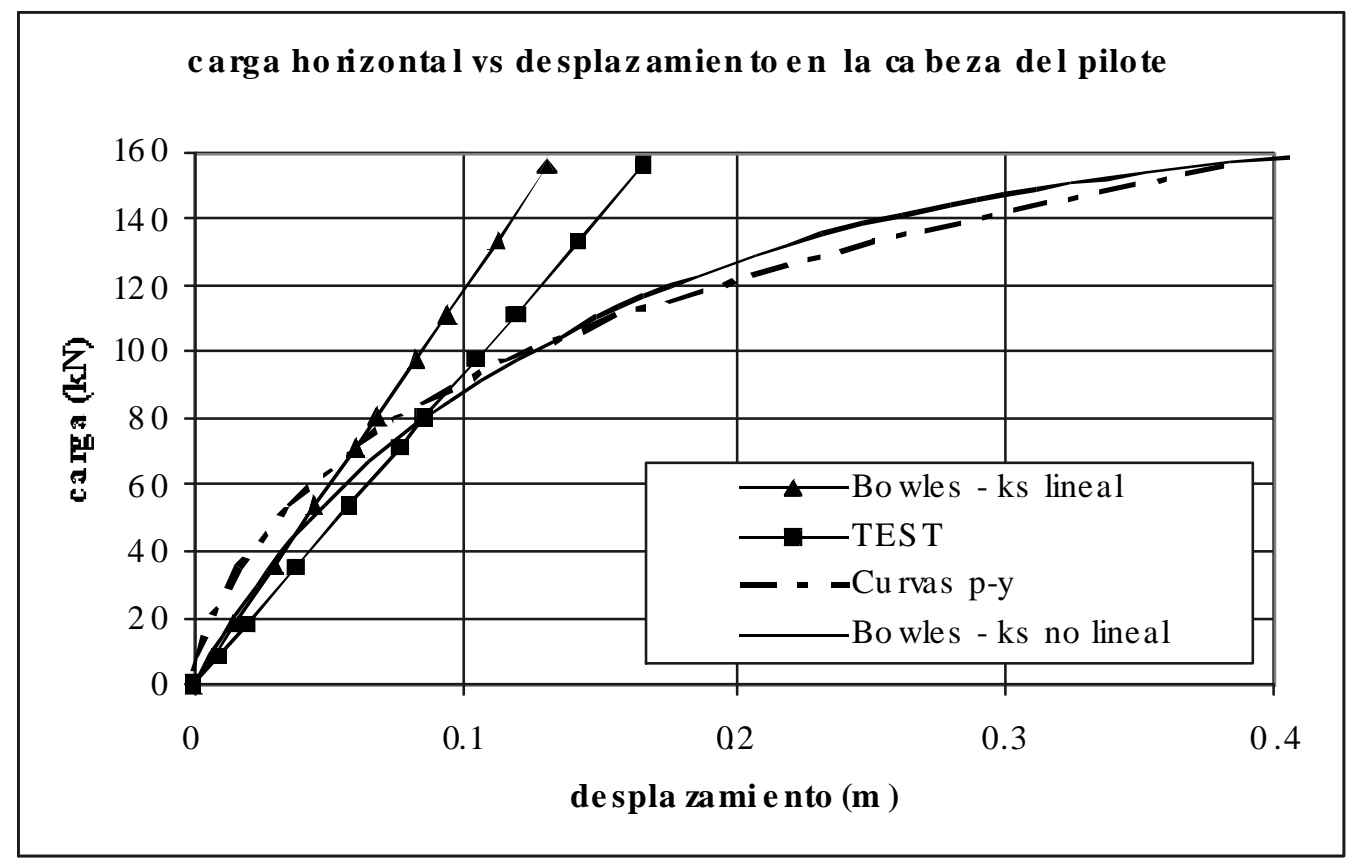

Figura 6. Desplazamiento en la cabeza vs carga lateral de la prueba de Matlock

Los resultados del método de curvas $p$-y también se ajustaron muy bien a los momentos y desplazamientos experimentales del ensaye de
Snyder (2004), (Figuras 7 y 8). Los momentos flexionantes tuvieron una diferencia máxima de $15 \%$ del momento máximo.

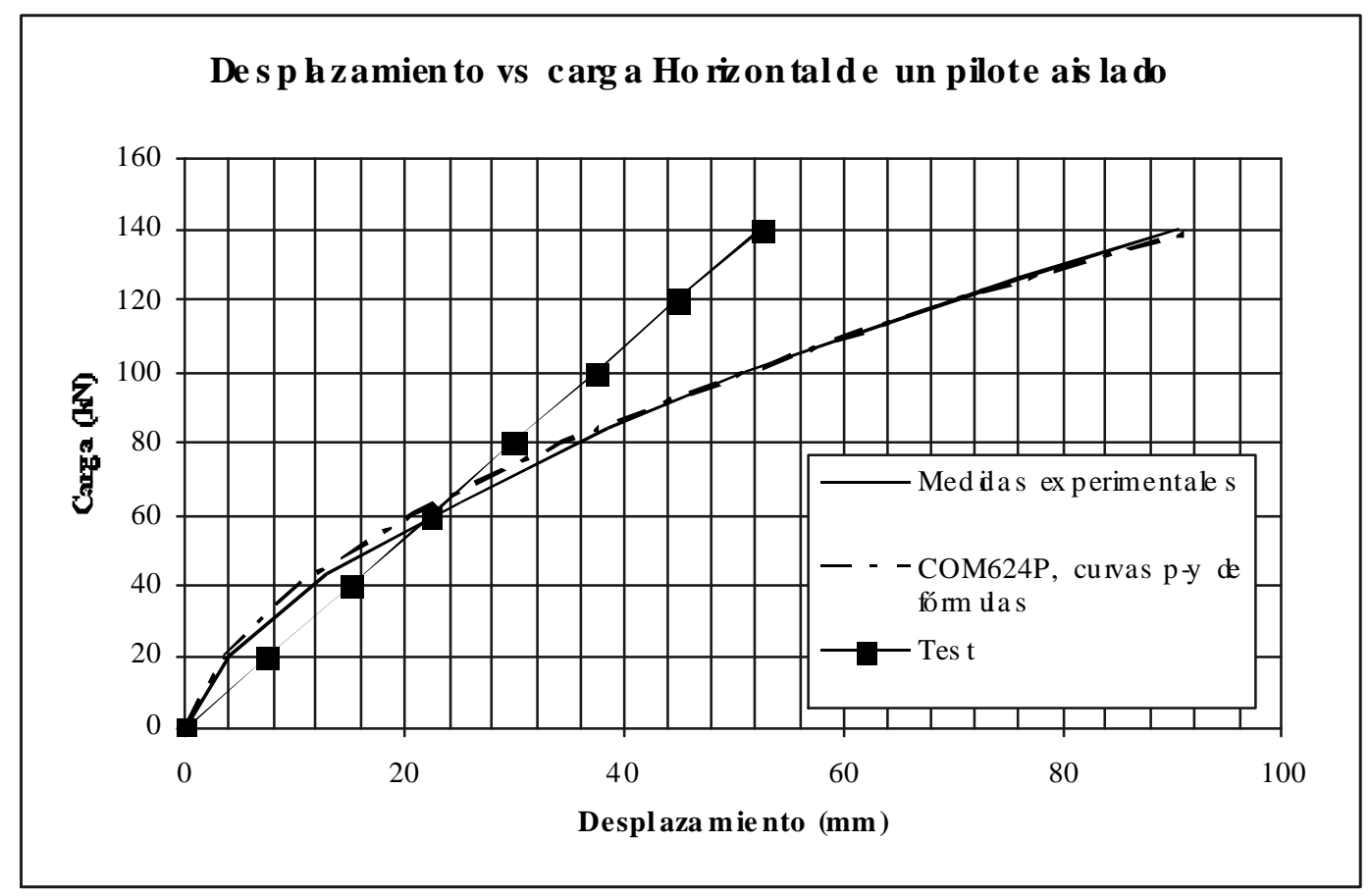

Figura 7. Desplazamiento vs carga lateral del ensaye de Snyder (2004) 


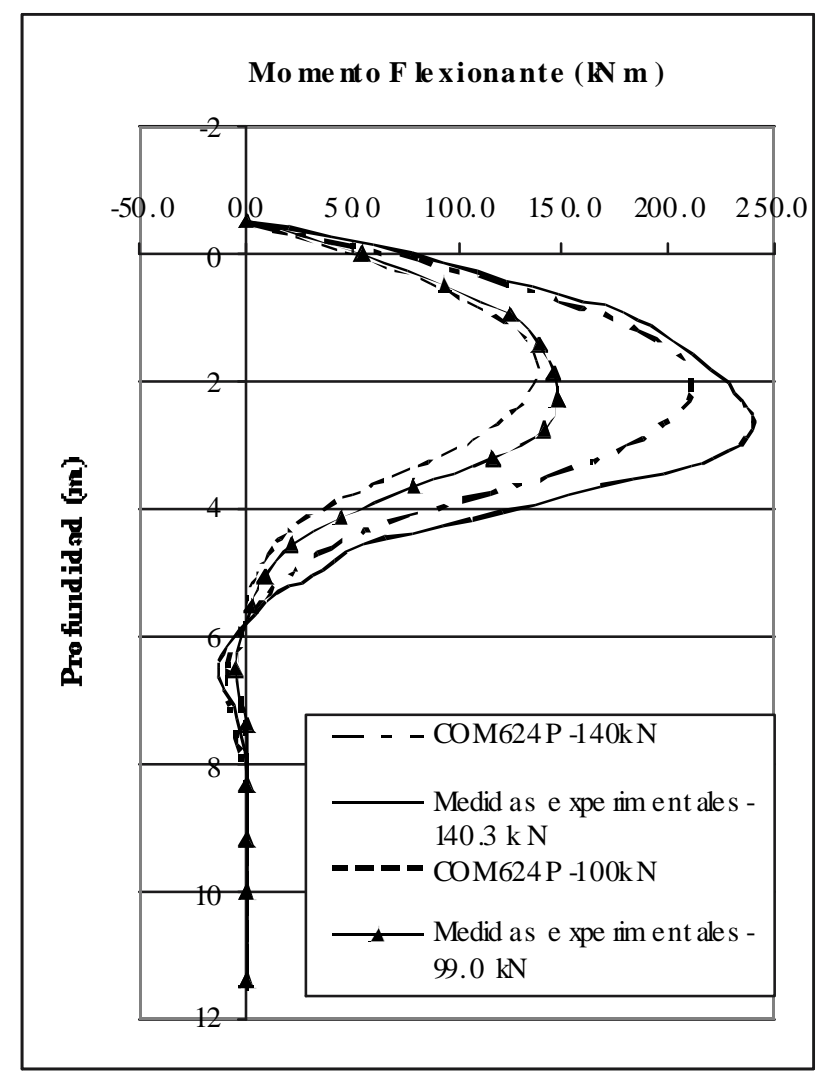

Figura 8. Perfiles de momentos flexionantes, experimentales y calculados de la prueba de Snyder (20 04)

Para tomar en cuenta el comportamiento no lineal del suelo se programó un procedimiento iterativo que calcula el módulo de reacciónks en función de la deformación unitaria del suelo. En la figura 6 podemos ver los desplazamientos calculados con el programa de Bowles. Aunque falta verificar este procedimiento en más pruebas de carga, en la prueba de Matlock proporcionó una mejoría notable en comparación con los resultados conseguidos considerando $k_{s}$ como parámetro lineal.

Los grupos de pilotes se analizaron con el método de curvas $p-y$ y elementos finitos. Para el primer método se usaron los multiplicadores de curvas $p-y$ propuestos por Rollins et al. (1998) y Snyder (2004). Cabe mencionar que aún hay diferencias importantes entre los multiplicadores propuestos hasta ahora, lo cual indica que hace falta la realización de mayor número de pruebas de carga para afinar los valores de estos multiplicadores. Además, los multiplicadores han sido obtenidos comúnmente de pruebas de carga donde la separación entre pilotes es igual en la dirección paralela $(S)$ y en la transversal $\left(S^{\prime}\right)$ a la carga lateral, pero en las gráficas de multiplicadores únicamente interviene la separación en sentido paralelo a la carga. Si por algún motivo $S^{\prime}$ fuera mayor que $S$ entonces los traslapes entre esfuerzos en el suelo provocados por los pilotes serían menores que cuando $S^{\prime}=S$, por lo que la eficiencia del grupo sería mayor y los multiplicadores resultarían conservadores. Si sucediera lo contrario, $S^{\prime}<S$, los esfuerzos en el suelo tendrían un mayor traslape, acercándolo más a la condición plástica y reduciendo la eficiencia del grupo; en esta situación, usar los multiplicadores hasta ahora calculados sería riesgoso. 
En la figura 9 se muestran las curvas de desplazamiento vs carga; por ejemplo, con un desplazamiento de $0.12 \mathrm{~m}$ la fila delantera soporta un promedio de $71 \mathrm{kN}$ mientras que la fila trasera un promedio de $53.4 \mathrm{kN}, 25 \%$ menor. Si entramos con estas cargas a la gráfica de momentos, figura 10, vemos que la fila delantera tiene un momento de $166 \mathrm{kN}-\mathrm{m}, 20 \%$ mayor que el de la fila trasera que tiene 135 $\mathrm{kN}-\mathrm{m}$. El momento flexionante del pilote aislado cargado con el promedio del grupo (62.3 $\mathrm{kN}$ por pilote) es aproximadamente $40 \%$ menor que el de la fila delantera. En la figura 11 se ilustran los perfiles de momentos flexionantes cuando la carga lateral en cada pilote es de 71.17 $\mathrm{kN}$. Debe notarse que aquí el mayor momento ocurre en los pilotes de la fila trasera, luego en los de la fila delantera y el menor momento se observa en el pilote aislado. Asimismo, la profundidad a la que ocurre el momento flexionante máximo va en aumento desde el pilote aislado hasta los pilotes de la fila trasera. Este hecho cobra importancia si consideramos que durante un sismo la estructura oscila y provoca que los pilotes que en un momento son delanteros, al cambiar de sentido el movimiento, se convierten en traseros, y los que son traseros ahora serán delanteros. Los pilotes deberán diseñarse teniendo en cuenta que los momentos máximos se pueden presentar a diferentes profundidades y habrá que reforzarlos en un tramo más amplio.

Los análisis de grupo realizados con elementos finitos TEST (se consideró al suelo elástico lineal) coinciden parcialmente con los resultados experimentales. En la figura 12 se muestra cómo el grupo de pilotes (filas trasera-FT, media-FM y delantera-FD) tienen un desplazamiento $50 \%$ mayor que el del pilote aislado, considerando que se le aplica una carga igual a la promedio del grupo, lo cual concuerda con los resultados experimentales de la figura 2.

Otra similitud entre cálculos y experimentos es la eficiencia de los grupos. La eficiencia de grupos de 6, 9 y 16 pilotes con separaciones de 3D se muestra en la figura 13. La tendencia indica que para grupos con más de 9 pilotes, $\eta$ se mantiene prácticamente constante y alrededor de $50 \%$, similar a la figura 4 de Ilyaset al . (2004).

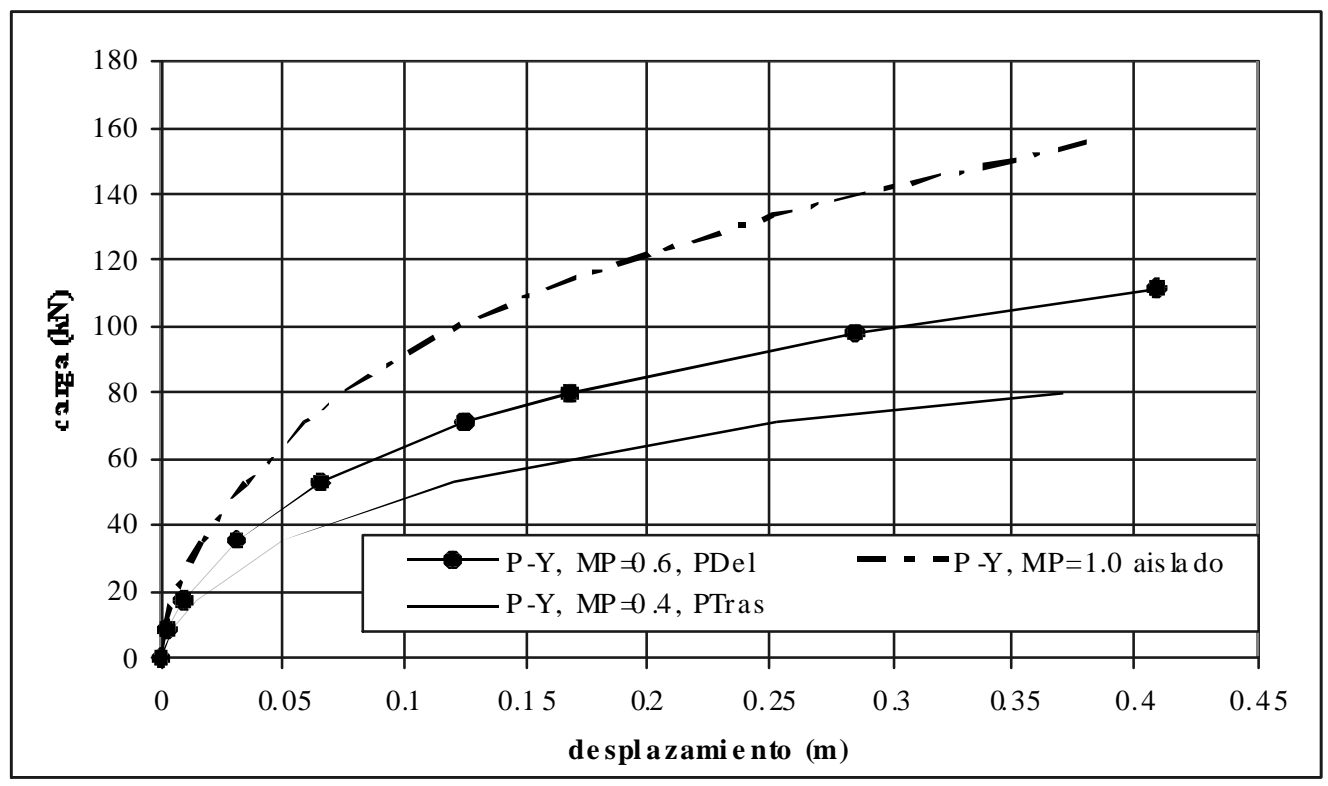

Figura 9. Análisis con método de curvas p-y. Desplazamiento en la cabeza vs carga lateral 


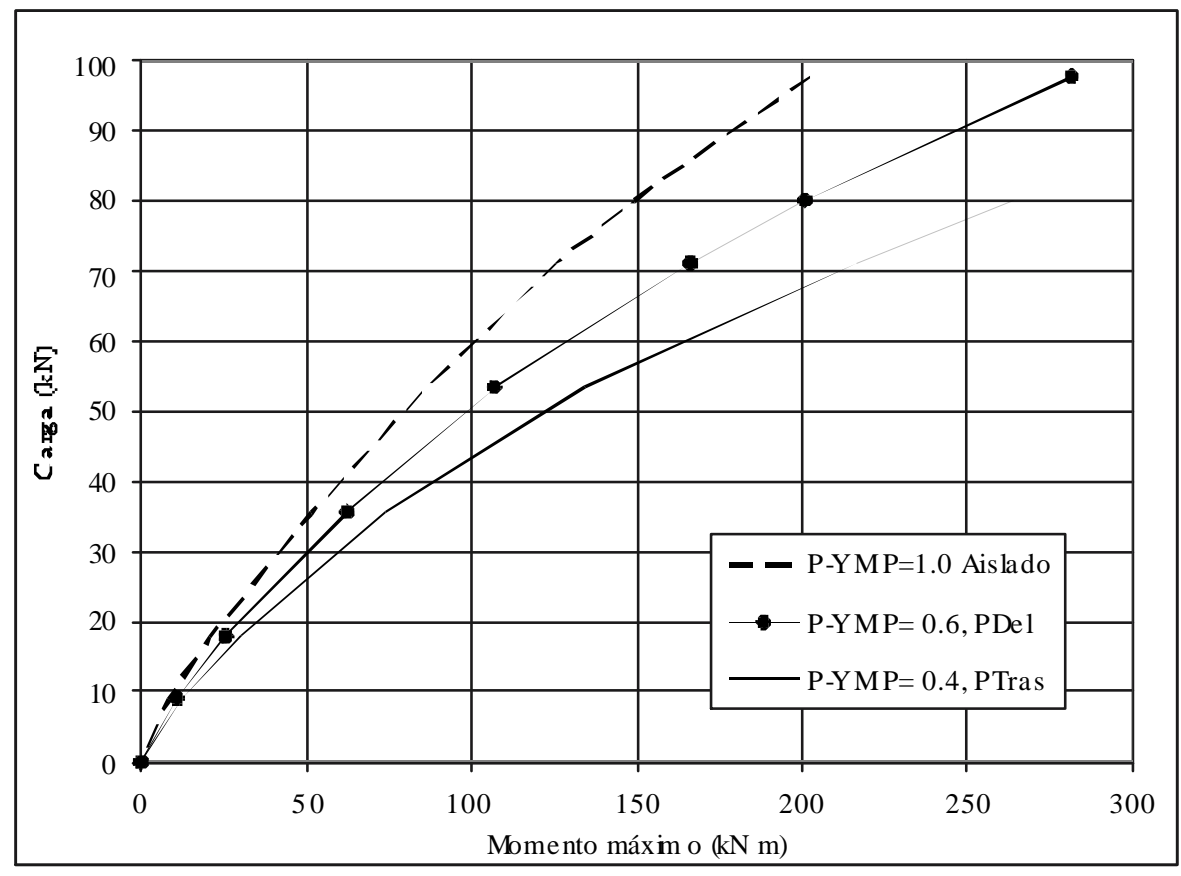

Figura 10. Análisis con método de curvas p-y. Momento flexionante máximo vs carga.

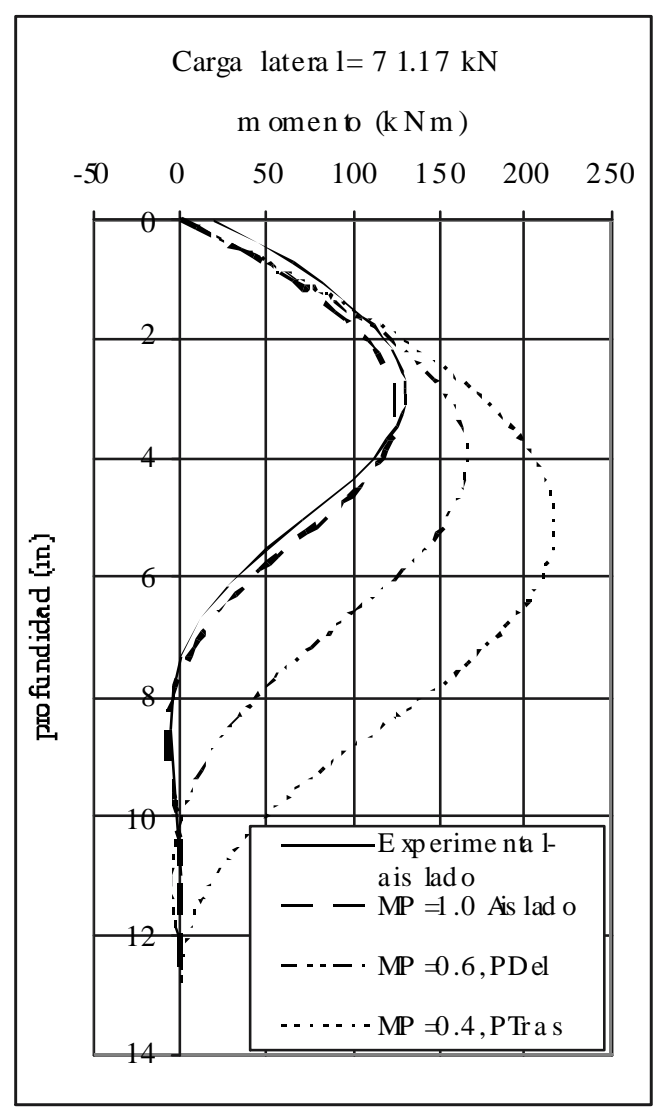

Figura 11. Perfiles de momentos flexionantes con carga lateral de $71.17 \mathrm{kN}$ por pilote 
Jaime-Paredes A. y Ruedas-Medina R.

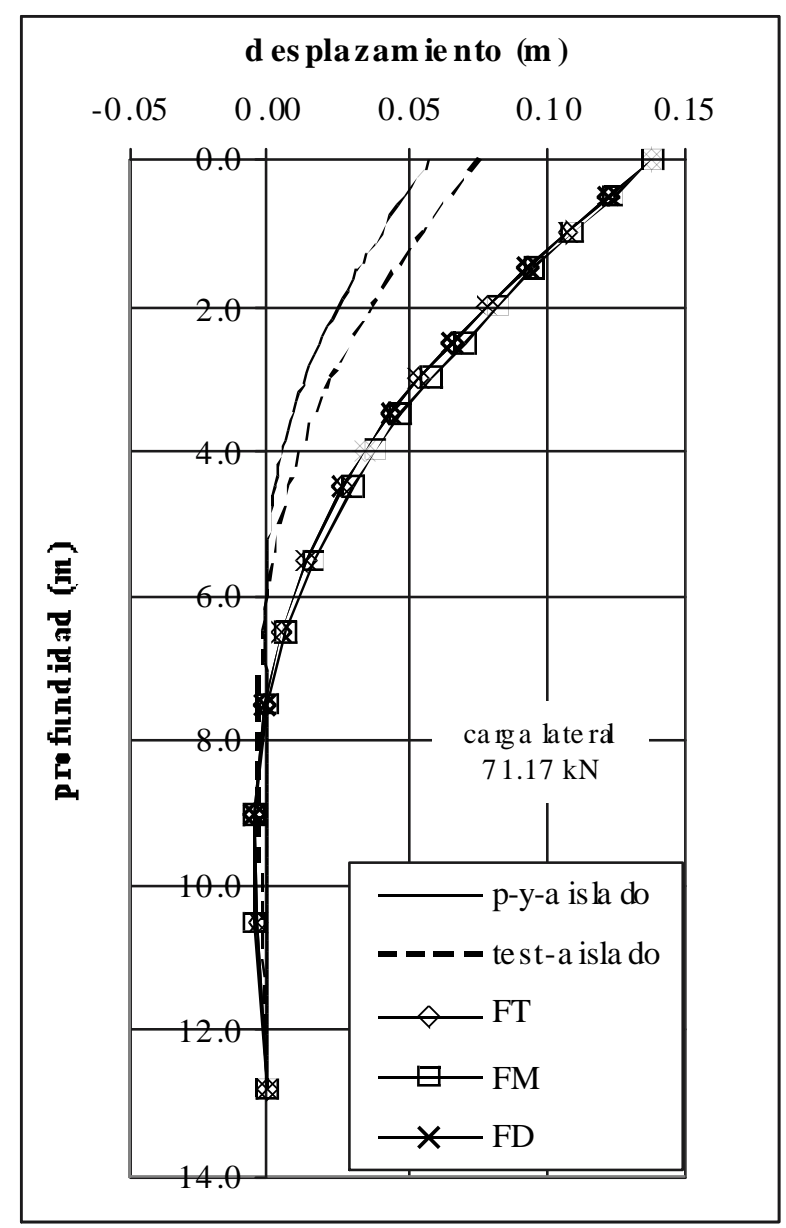

Figura 12. Perfil de desplazamientos. Análisis de grupo de pilotes con elementos finitos

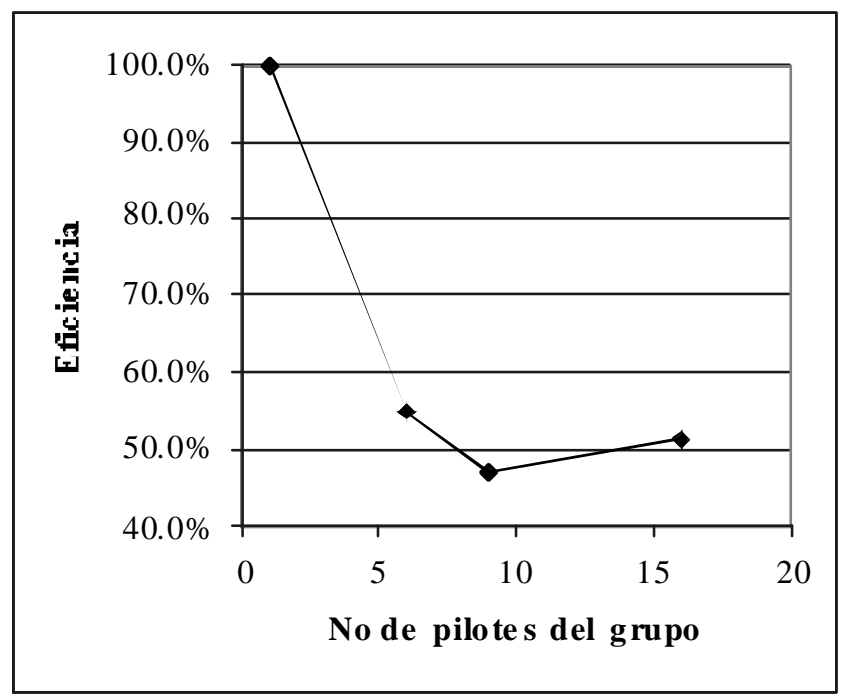

Figura 13. Eficiencia de grupos de pilotes 
Sin embargo, se encontró que los momentos flexionantes eran iguales en las filas delantera y trasera, un resultado que es completamente diferente a los experimentales mostrados en la figura 3.

Por otra parte, para confirmar que la eficiencia de un grupo depende no sólo de la separación entre pilotes, del número y del nivel de carga alcanzado, sino también de la distribución o configuración que tengan, se hicieron dos análisis con grupos de 14 y 16 elementos con igual carga promedio pero distinta configuración dentro de una misma área, figura 14. Por los resultados antes vistos, la eficiencia de estos dos grupos debería ser muy parecida; sin embargo se encontró que la eficiencia del grupo de 14 pilotes es $79 \%$, más alta que el $51 \%$ del grupo de 16. Como puede inferirse, para mejorar el funcionamiento de la cimentación se debe buscar la distribución más adecuada.

Asimismo, las gráficas de eficiencia nos demuestran que aumentar el número de pilotes no significa un aumento proporcional de la capacidad de carga de la cimentación.

Todo parece indicar que el aumento de eficiencia se debe a que hay un menor traslape de esfuerzos generados enfrente y detrás de los pilotes. Se deduce que la carga lateral más peligrosa es la que tiene una dirección paralela a las filas o hileras de pilotes, como la mostrada en la figura 14 a) o las cargas indicadas por las flechas grises de $14 \mathrm{~b}$ ). Se observa que el análisis lineal no modela la dependencia que hay entre eficiencia y desplazamiento del grupo, como se puede concluir de las gráficas de desplazamiento vs carga lateral, figura 15.

Cabe comentar que los resultados del a nálisis con elementos finitos dependen del grado de refinamiento que tenga la malla. En este estudio la eficiencia de grupo tuvo un aumento de hasta $10 \%$ cuando se refinaba la malla alrededor de los pilotes. Aunque en los párrafos anteriores se reporta la menor eficiencia calculada, esta variación no debe perderse de vista y sería recomendable profundizar al respecto.
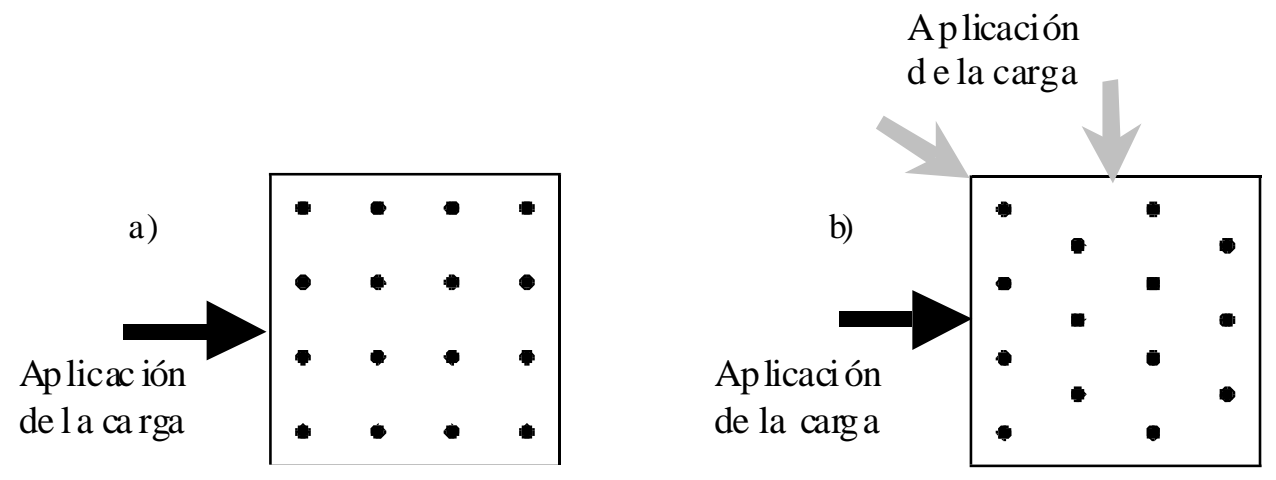

Figura 14. Configuración de grupos de 16 y 14 pilotes. 


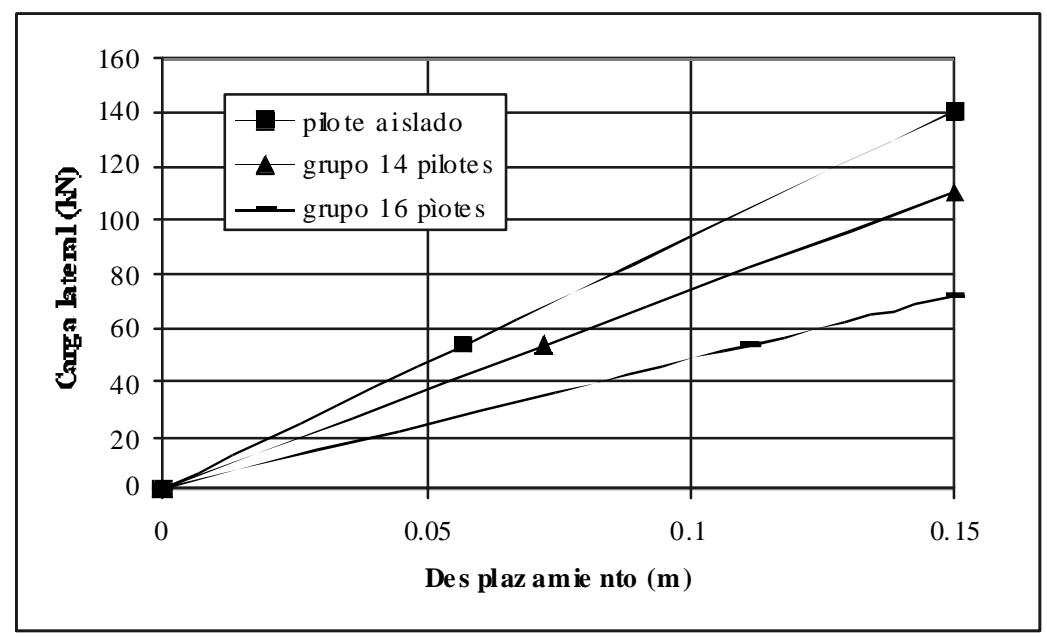

Figura 15. Desplazamiento vs carga de grupos de pilotes con diferente distribución

\section{Conclusiones}

El estudio del comportamiento de pilotes revela que los pilotes aislados son más eficientes ante carga lateral que los pilotes actuando en un grupo. Se llegan a tener eficiencias de hasta 50\% en el caso de grupos grandes de pilotes.

Dentro del grupo hay una distribución diferenciada de cargas por filas. La fila delantera es la que soporta la mayor cantidad de carga, de 75 a $100 \%$ de lo que soporta un pilote aislado con el mismo desplazamiento. Las filas media y trasera soportan entre 50 y $75 \%$ de lo de un pilote aislado. Es decir, en una cimentación real no basta agregar pilotes para resistir la carga lateral, sino que hay que revisar el comportamiento del grupo teniendo especial cuidado en la distribución de los pilotes (geometría en planta) y su separación centro a centro.

De los tres métodos empleados, el método de curvas $p-y$ es el que mejor modela el comportamiento experimental, tanto en pilotes aislados como en grupos y la distribución de cargas que se presentan en estos. No obstante, debe cuidarse que la separación entre pilotes en el sentido perpendicular a la carga sea mayor o igual a la separación en la dirección paralela, para que el uso de multiplicadores de curvas $p$-y esté del lado seguro.

El programa de Bowles modela linealmente la respuesta de la interacción pilote suelo y proporcionan resultados satisfactorios cuando la carga lateral entra en un rango de cargas reducido; sin embargo, el programa mejora cuando se toma en cuenta el comportamiento no lineal del suelo. Por su parte, con el método de elementos finitos-TEST-elástico lineal se pudo deducir que la carga más desfavorable, y con la cual se deben hacer los a nálisis, es la que tiene dirección paralela a las hileras de pilotes. Con los elementos finitos también se encontró que la eficiencia depende no sólo de la separación, número de pilotes y desplazamiento alcanzado, sino también de la distribución de pilotes, misma que influye en la distribución de esfuerzos en el suelo. Mientras más cercanía tengan los pilotes y estén alineados con la carga, mayores serán los esfuerzos en el suelo y la resistencia lateral de estos elementos disminuye.

De este esudio también se infiere la importancia de analizar la interacción entre estructuras piloteadas contiguas. 


\section{Referencias}

Bowles J. (1974). Analytical and Computer Methods in Foundation Engineering. McGraw-Hill, Nueva York, EU, pp. 519.

Brown D., Reese L. y O'Neill M. (1987). Cyclic Lateral Loading of a Large-Scale Pile Group. Journal of Geotechnical Engineering, Vol. 113, No. 11, pp. 1326-1343.

Brown D., Morrison C. y Reese L. (1988). Lateral Load Behavior of Pile Group in Sand. Journal of Geotechnical Engineering, Vol. 114, No. 11, pp.1261-1276.

Hetenyi M. (1946). Beams on Elastic Foun da tions. Michigan, University of Michigan Press.

Ilyas T., Leung C.F., Chow Y.K. y Budi S.S. (2004). Centrifuge Model Study of Laterally Loaded Pile Groups in Clay. Journal of Geotechnical and Geoenvironmental Engineering, Vol. 130, No. 3, pp. 274-283.

Li Liu Xiangyue, Romo M. y Magaña R. (1992). TEST92: Un programa de computadora para simular excavaciones en suelos elasto-plásticos. Informe interno Instituto de Ingeniería, UNAM, México.

Matlock H. (1970). Correlations for Design of Laterally Loaded Piles in Soft Clay,
OTC 1204. Second Annual Offshore Technology Conference, Dallas, Texas, abril.

Poulos H.G. (1971a). Behavior of Laterally Loaded Piles: I-Single Piles. Journal of Soil Mechanics and Foundations Division, Vol. 97, No SM5, pp. 711-732.

Poulos H.G. (1971b). Behavior of Laterally Loaded Piles: II-Pile Groups. Journal of Soil Mechanics and Foundations Division, Vol. 97, No SM5, pp. 733-751.

Randolph M.F. (1981). The Response of Flexible Piles to Lateral Loading. Géotechnique 31, No 2, pp. 247-259.

Rollins K., Peterson K. y Weaver T. (1998). Lateral Load Behavior of Full-Scale Pile Group in Clay.Journal of Geotechnical and Geoenvironmental Engineering, Vol. 6, pp. 468-478.

Snyder J. (2004). Full-Scale Lateral-Load test of a 3X5 Pile Group in Soft Clays and Silts. Tesis de maestría en ingeniería, Brigham Young University, EU.

Wang S.T. y Reese L. (1993). User Guide: Laterally Loaded Pile Analysis Program for the Microcomputer, Version 2.0, Ensoft Inc, Austin Texas, EU.

\section{Semblanza de las autores}

Alberto Jaime-Paredes. Estudió la licenciatura, maestría y doctorado en la Universidad Nacional Autónoma de México. Realizó estudios de posgrado en la Universidad de Pordue, EU. En 1975, comenzó su labor como investigador en el Instituto de Ingeniería de la UNAM. Ha publicado más de 90 artículos técnicos en revistas extranjeras y nacionales, además, ha colaborado en memorias de congresos en el país y en el extranjero. Por diez años fue servidor público con cargos de mando medio supe rior en la CFE y la CNA. Actualmente es jefe de la División de Ingenierías Civil y Geomática de la FI de la UNAM.

Roberto Ruedas-Medina. Estudió la licenciatura y obtuvo el grado de maestro con la tesis Comportamiento de grupos de pilotes bajo carga lateral en suelo blando, ambos en la Facultad de Ingeniería de la UNAM. Colaboró en el desarrollo de los Planes Maestros Regionales sobre Infraestructura Inmobiliaria del IMSS. Haparticipado en los análisis geotécnicos de presas de tierra y enrocamiento. 